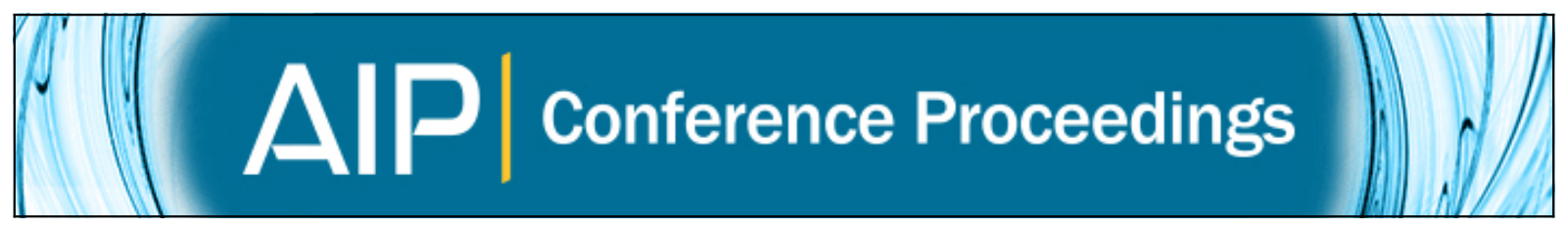

\title{
A tapered box model of the cochlea
}

Luyang Sun, Guangjian Ni, and Stephen Elliott

Citation: AIP Conference Proceedings 1703, 070014 (2015); doi: 10.1063/1.4939388

View online: http://dx.doi.org/10.1063/1.4939388

View Table of Contents: http://scitation.aip.org/content/aip/proceeding/aipcp/1703?ver=pdfcov

Published by the AIP Publishing

\section{Articles you may be interested in}

Finite element cochlea box model - Mechanical and electrical analysis of the cochlea

AIP Conf. Proc. 1703, 070012 (2015); 10.1063/1.4939386

Four-chamber cochlea box model: Establishing acoustic comfort, illustrating injury and toward therapy.

J. Acoust. Soc. Am. 129, 2382 (2011); 10.1121/1.3587726

Mechanical cochlear-partition parameter variation in a box-cochlea model

J. Acoust. Soc. Am. 100, 2630 (1996); 10.1121/1.417736

Global and local parameter and response attributes in a box-cochlea model

J. Acoust. Soc. Am. 95, 2840 (1994); 10.1121/1.409613

Effects of scalae tapering, perilymph viscosity, helicotrema, and the cochlear map function (CMF) on models of the cochlea

J. Acoust. Soc. Am. 84, S54 (1988); 10.1121/1.2026362 


\title{
A Tapered Box Model of the Cochlea
}

\author{
Luyang Sun, Guangjian Ni and Stephen Elliott
}

\author{
Institute of Sound and Vibration Research, University of Southampton, Highfield Campus, Southampton, UK
}

\begin{abstract}
The complicated, three dimensional geometry of the fluid chambers in the cochlea is often represented in models of its mechanics by a box with a uniform area along its length. In this paper we use previous measurements of the variation in area of the two fluid chambers along the length of the cochlea in various mammals, to calculate the variation in the "effective area" that determines the 1D fluid coupling, which is given by the harmonic mean of the two chamber areas. The square root of this effective area is found to vary surprisingly linearly along the cochlea length in several mammalian species. This suggests a variation of the box model in which the width and height of the two fluid chambers are still equal, but now decrease linearly along its length. The width of the basilar membrane, BM, is assumed to increase linearly along the length of the model. The analytic form of the $1 \mathrm{D}$ fluid pressure distribution due to elemental BM motion is derived for this tapered box model. The added mass due to the near field acoustic coupling can also be computed, which surprisingly turns out to be almost constant along the length of the BM. The coupled response of the box model with a passive BM can then be readily calculated. Although the pressure distributions due to elemental fluid coupling are very different in the uniform and tapered box models, the distribution of the passive BM response in the coupled models are very similar in the two cases, although the overall level of the response in the tapered model is about $10 \mathrm{~dB}$ greater than that in the uniform model.
\end{abstract}

\section{INTRODUCTION}

The mammalian cochlea has a complicated, three dimensional, geometry including coiling [10]. Its underlying dynamics, however, can be illustrated with an uncoiled box model [3, 7], which is generally assumed to have a uniform cross section along its length as shown in the left part of Fig. 1. If the uniform box model represents a first approximation to the cochlear geometry, for the calculation of the fluid coupling, a sensible second approximation would thus appear to be the tapered box model, as shown in the right part of Fig. 1. There are several sources of information about the variation in the physical dimensions of the fluid chambers, along the length of the cochlea in different species, of which the database of Salt [12] is perhaps the most comprehensive.

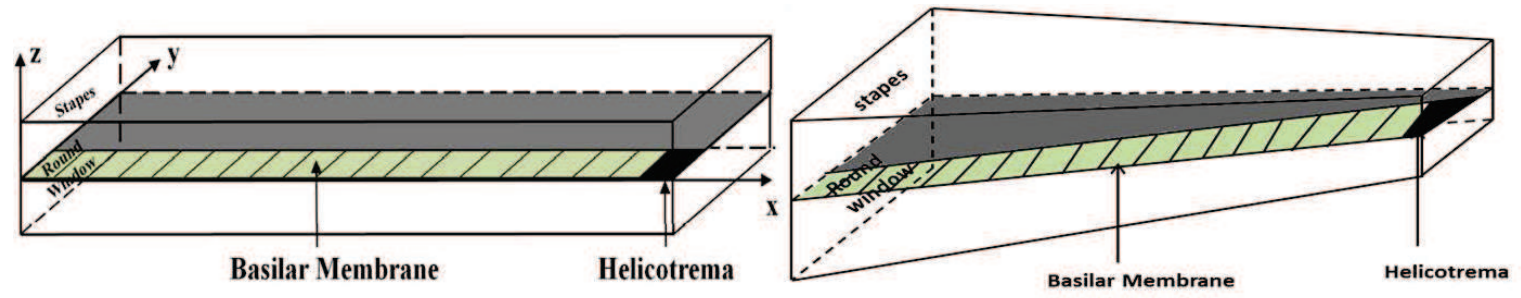

FIGURE 1. Left panel: the uniform box model of the cochlea, in which the height of the fluid chamber and the width of the cochlear partition and basilar membrane are constant along the length. Right panel: the tapered box model, in which the cross sectional area has square shape, but decrease along the length, the width of the basilar membrane increases along the length.

The mechanics of the cochlea can be thought of as an interaction between the fluid coupling along these chambers and the dynamic behavior of the cochlear partition that separates them. The fluid coupling can itself be divided into two components [4], one due to the plane wave component of the pressure in each chamber, known as the 1D, or far field fluid component, and one due to the total pressure variations close to the BM, also known as the near field component, which, together with the plane wave go to make up the full 3D fluid coupling. The 1D component of the fluid coupling depends on the variation of a single "effective area" along the length of the cochlea [4, 9], which is a function of the areas of the two physical fluid chambers, above and below the BM. The near field component of the fluid coupling, on the other hand, contributes an added mass to the dynamics of the BM, which depends on the width and position of the $\mathrm{BM}$ as well as the variation of the geometrical size of the fluid chamber. Since the near field pressure distribution is local to the BM, whose width is small compared with that of the cochlear partition over most of the cochlear length, therefore, this pressure is assumed not to be strongly dependent on the area of the fluid chambers [4]. 


\section{THE VARIATION OF THE COCHLEAR CHAMBER CROSS-SECTIONAL AREA AND THE BM WIDTH FROM MEASUREMENT DATA}

The geometrical feature that is important in the 1D fluid coupling is the effective area of each chamber, defined by $[4,9]$ to be

$$
A_{e}(x)=\frac{2 A_{1}(x) A_{2}(x)}{A_{1}(x)+A_{2}(x)},
$$

where $x$ is the longitudinal variable and $A_{1}(x)$ and $A_{2}(x)$ are the area in the upper and lower fluid chambers. Although $A_{1}(x)$ and $A_{2}(x)$ vary in a complicated way with position, as shown in Fig. 2 for the guinea pig [12], the variation of $A_{e}(x)$, and particularly, the square root of this effective area is much smoother. The latter can be reasonably well approximately by a linear longitudinal variation in this case, as shown in Fig. 3. The linearized variation is a reasonable fit to the square root of the effective area, although it underestimates the true value by about $11 \%$ near the base.
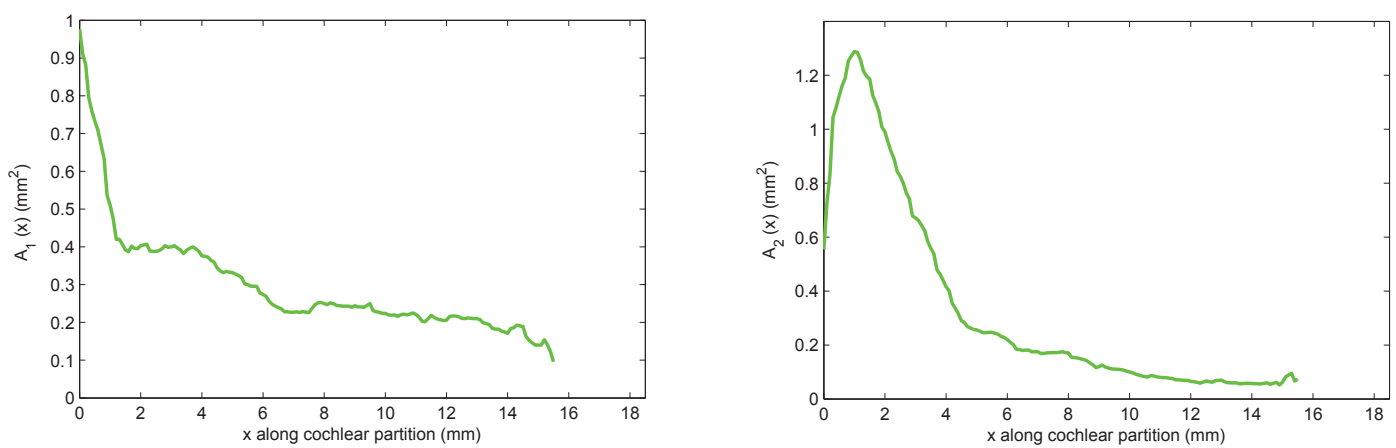

FIGURE 2. $A_{1}(x)$ and $A_{2}(x)$ for the guinea pig [12, 13].

The least square method has been used to fit a linear variation to the square root of the effective cochlear chamber cross section area in a number of mammalian species (the width and height of fluid chamber along the cochlear partition, which are assumed to be equal), as shown in the left panel of Fig. 4. The linear curve is assumed to extend over the entire length of the cochlea, $18.5 \mathrm{~mm}$ in the guinea pig, even though the experimental measurements of Salt [12] only extended to about $16 \mathrm{~mm}$. The effective width of the tapered fluid chamber in guinea pig and cat cochlea, which is given as $W_{e}=H_{e}=\sqrt{A_{e}}$, has also been constrained to be no less than the BM width at the apex. The assumed variation of the BM width measured along the length of the cochlea for five different species is shown in the right panel of Fig. 4.
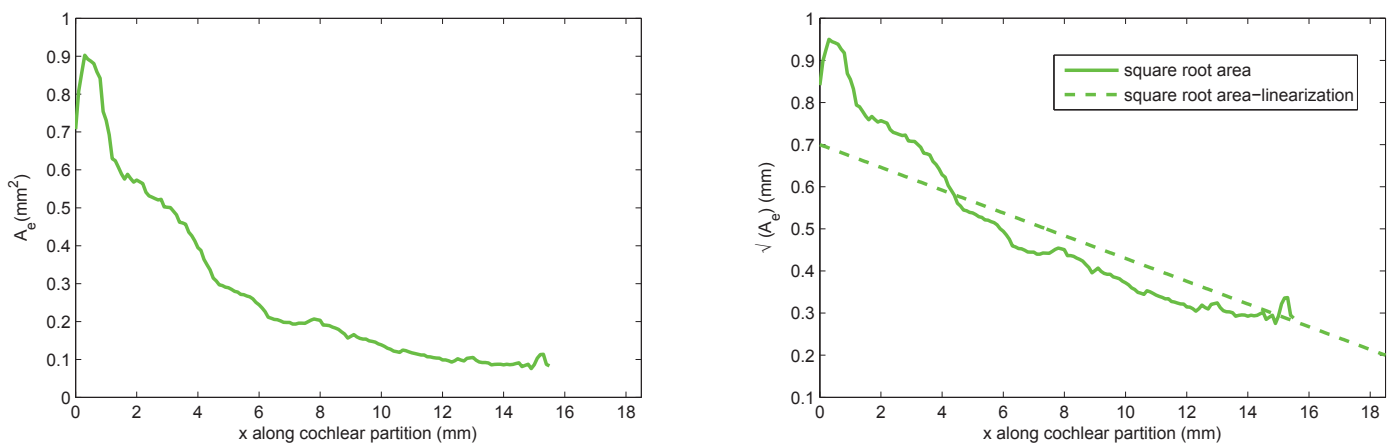

FIGURE 3. Effective area (left panel) of cochlear chamber for the guinea pig and its square root (right panel) (solid line for $\sqrt{A_{e}}$ and dashed line for linearized $\sqrt{A_{e}}$ ). 

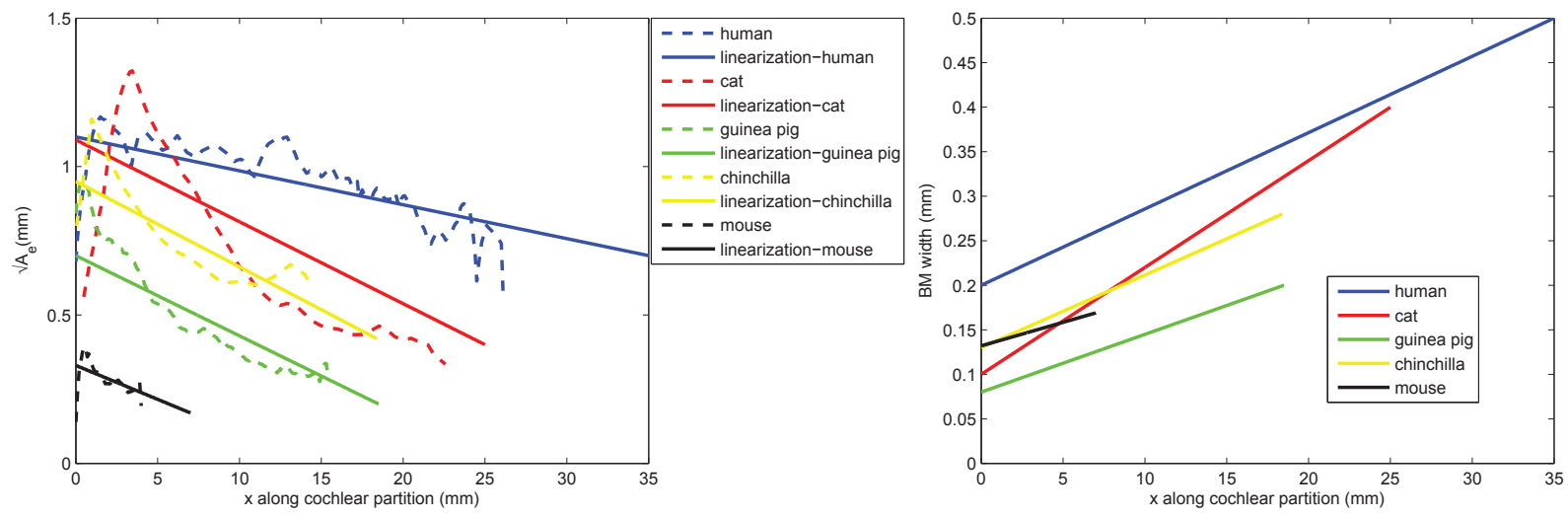

FIGURE 4. Left panel: Variation of square root of the effective cochlear chamber cross-sectional area along the cochlear partition for five species (solid line for $\sqrt{A_{e}}$, dashed line for $W_{l}$ and $H_{l}$, where $W_{l}$ and $H_{l}$ are the linearized variation of $W_{e}$ and $H_{e}$ by using least square method) calculated from the measured $A_{1}(x)$ and $A_{2}(x)$ in Salt [12], and the assumed linear variations of $W_{e}(x)$ and $H_{e}(x), W_{l}(x)$ and $H_{l}(x)$ (the square root of effective cochlear cross-sectional area), along the whole cochlear partition for five species. Right panel: Linear approximation to the variation of the BM width, along the cochlear partition in five species. The BM width for human is derived from [4], cat from [1], guinea pig from [5], chinchilla from [2], and mouse from [6].

\section{FLUID COUPLING}

\section{D Pressure and Full 3D Pressure}

The far field pressure component of the pressure in each of the fluid chambers is derived by one dimensional analysis for a box model of the cochlea with tapered asymmetric fluid chambers, as described by $\mathrm{Ni}$ [8], as

$$
\begin{gathered}
\left.p_{F}(n)\right|_{0<n<n_{0}-1}=-16 i \omega \rho \Delta^{2} v_{0} \sqrt{\frac{B_{l}\left(n_{0}\right) W_{l}\left(n_{0}\right) B_{l}(n)}{W_{l}(n)}} \sum_{n^{\prime}=n_{0}}^{N} \frac{1}{H_{l}^{2}\left(n^{\prime}\right)}, \\
\left.p_{F}(n)\right|_{n_{0}<n<N}=-16 i \omega \rho \Delta^{2} v_{0} \sqrt{\frac{B_{l}\left(n_{0}\right) W_{l}\left(n_{0}\right) B_{l}(n)}{W_{l}(n)}} \sum_{n^{\prime}=n}^{N} \frac{1}{H_{l}^{2}\left(n^{\prime}\right)},
\end{gathered}
$$

where $n_{0}=x_{0} / \Delta$, which is the element vibration at position $x_{0}$ of width $\Delta, \Delta=L / N$. Eq. (2) can be used to calculate the far field contribution to the pressure difference in the tapered cochlea due to asymmetry in the two fluid chambers. However, for the uniform box model, Eq. (2) can be simplified into

$$
\begin{gathered}
\left.p_{F}(x)\right|_{0<x<x_{0}-\Delta}=2 i \omega \rho \Delta \frac{\left(L-x_{0}\right)}{h_{a v}} v_{0}, \\
\left.p_{F}(x)\right|_{x_{0}<x<L}=2 i \omega \rho \Delta \frac{(L-x)}{h_{a v}} v_{0},
\end{gathered}
$$

where $h_{a v}$ is the average value of the effective height, equal to $\pi^{2} W_{a v} H_{a v} / 8 B_{a v}, W_{a v}, H_{a v}$ and $B_{a v}$ are the average values of $W_{l} H_{l}$ and $B_{l}$. Besides considering the far field pressure, the near field pressure should be added to calculate the full 3D pressure. it is the full 3D pressure, which is as shown in Fig. 5, using inverse Fourier transform of its wavenumber description, as described by Elliott et al. [4].

\section{Near Field Fluid Loading}

Apart from the physical mass per unit area of the BM, fluid loading will provide some additional mass, whose variation is affected by the values of $H_{l}(x), W_{l}(x)$ and $B_{l}(x)$. If $W_{l}(x)=H_{l}(x)$, as assumed here and $\beta(x)$ is equal to $B_{l}(x) / W_{l}(x)$, the effective additional thickness of BM due to the fluid coupling given by [4] can be written as

$$
T_{f}(x)=H_{l}(x)\left(\frac{8}{3 \pi^{2}} \beta(x)+\sum_{n=1}^{\infty} \frac{4}{n \pi^{3}} \beta(x) \operatorname{coth}(n \pi)\left[\frac{1+\cos (n \pi \beta(x))}{1-n^{2} \beta^{2}(x)}\right]^{2}\right) .
$$




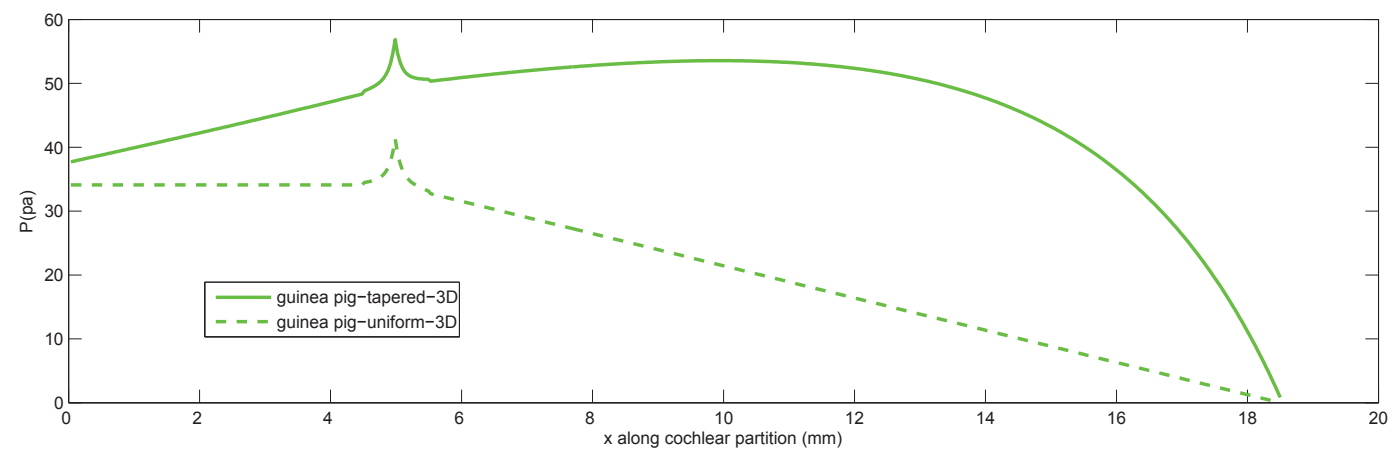

FIGURE 5. The calculated variation of the modal pressure difference, including both far and near fluid components, for the uniform and tapered box models of the guinea pig cochlea, when only a single element of the discrete BM at $x=5 \mathrm{~mm}$ is driven sinusoidally with a velocity of $10 \mathrm{~mm} \mathrm{~s}^{-1}$ at a frequency of $1 \mathrm{kHz}$.

It is interesting that although the effective thickness changes considerably if either $H$ or $B / W$ varies independently, as shown in the left panel of Fig. 6, the combination of their variation in the tapered box model gives an effective thickness which is more independently of the position along the cochlea. The added mass due to near field fluid coupling turns out to be surprisingly constant along the length of the BM, as shown in the right panel of Fig. 6.
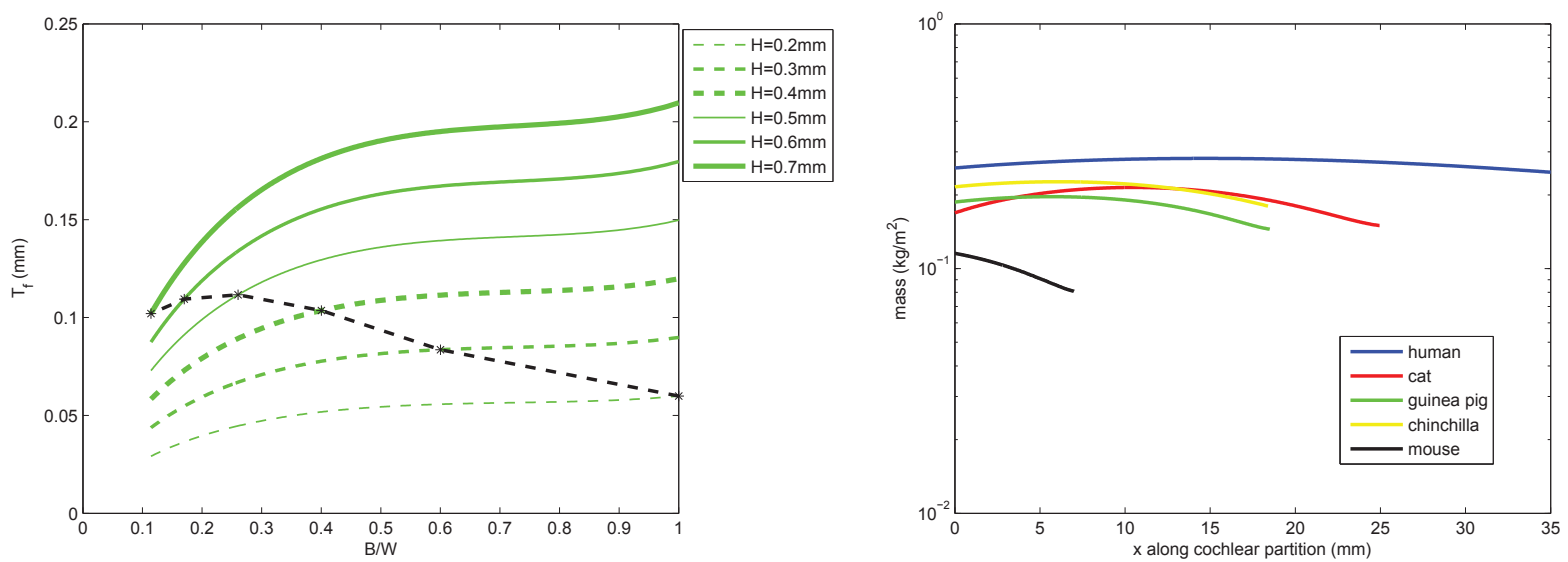

FIGURE 6. Left panel: effective thickness of fluid due to near field loading as a function of $B_{l} / W_{l}$ for various values of $H_{l}$. The stars and black dashed line correspond to the values of $B / W$ as $H$ varies along the length of the guinea pig cochlea for the tapered box model. Right panel: Variations of the total mass along the cochlear partition, including fluid added mass, for five species.

\section{COUPLED COCHLEAR RESPONSE}

The coupled mechanics of the cochlea from the box model can be calculated using an elemental approach [4], in which the fluid coupling and BM admittance are combined in a matrix formulation. The fluid coupling includes the variation in fluid chamber area and BM width described above, and the BM admittance is assumed to be passive. Figure 7 shows that the calculated distribution of the coupled BM velocity in the tapered and uniform box model including 3D fluid coupling. In the uniform box model, $W, H$, and $B$ are taken to be the average of those used in the tapered box model. The tapered box model has a peak velocity which is about $10 \mathrm{~dB}$ greater than the uniform box model and a somewhat faster roll off at high frequencies. 

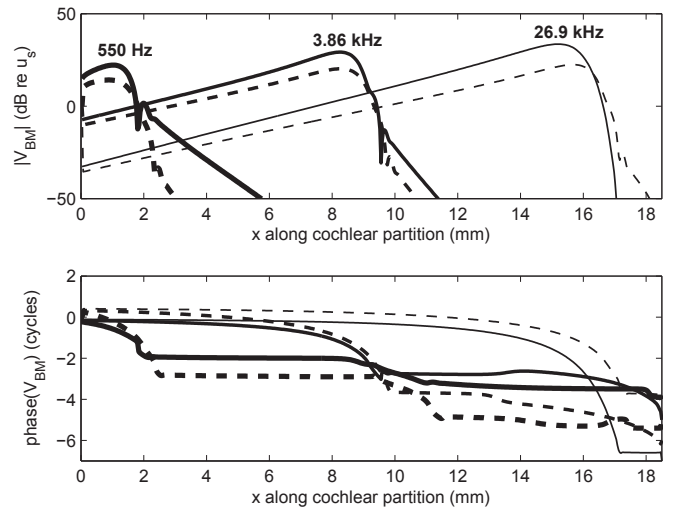
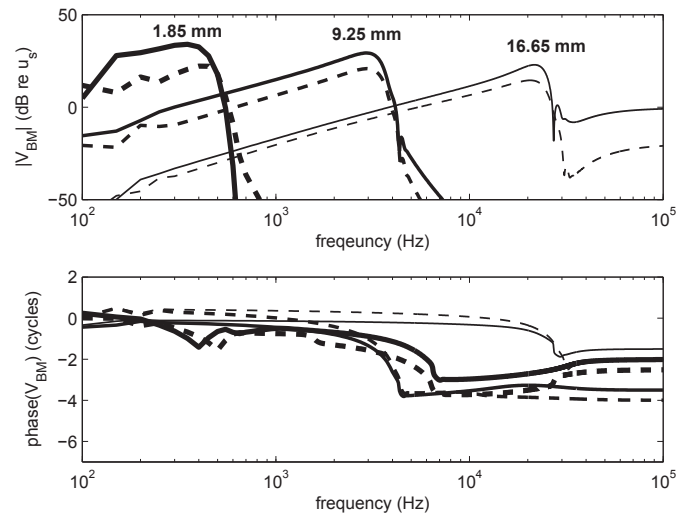

FIGURE 7. The magnitude and phase of BM transverse velocity along cochlear partition as a function of position at three frequencies and as a function of frequency at three positions in 3D uniform and tapered box model for the guinea pig cochlea. Solid lines for 3D tapered model, dashed lines for 3D uniform model.

\section{CONCLUSION}

A simple modification of the widely used box model can be used to take into account the observed longitudinal variation of effective fluid chamber area and BM width. This tapered box model gives an added BM mass, due to the fluid coupling, which decreases along the cochlea. The passive coupled response is similar for the uniform and tapered box models although the peak BM velocity is increased by about $10 \mathrm{~dB}$ in the case of the tapered model.

\section{ACKNOWLEDGMENTS}

This research is supported by EU project SIFEM (Grants FP7-600933) on the multi-scale modelling and 3D visualization for the inner-ear.

\section{REFERENCES}

[1] Cabezudo LM (1978) The ultrastructure of the basilar membrane in the cat. Acta Otolaryngol 86:160-175

[2] Dallos P (1970) Low-frequency auditory characteristics: species dependence. J Acoust Soc Am 78:489-499

[3] de Boer E (1991) Auditory physics. Physical principles in hearing theory III. Phys Rep 203:125-231

[4] Elliott SJ, Lineton B, Ni G (2011) Fluid coupling in a discrete model of cochlear mechanics. J Acoust Soc Am 130:1441-1451

[5] Fernandez C (1952) Dimensions of the cochlea (Guinea pig). J Acoust Soc Am 24:519-523

[6] Keiler S, Richter CP (2001) Cochlear dimensions obtained in hemicochlea of four different strains of mice: CBA/CaJ, 129/CD1, 129/SvEv and C57BL/6J. Hear Res 162:91-104

[7] Neely ST (1985) Mathematical modelling of cochlear mechanics. J Acoust Soc Am 78:345-352

[8] Ni G (2012) Fluid coupling and waves in the cochlea. University of Southampton, Faculty of Engineering and the Environment, Doctoral Thesis, p. 215

[9] Peterson LC, Bogert BP (1950) A dynamical theory of the cochlea. J Acoust Soc Am 22:369-381

[10] Pickles JO (2008) An Introduction to the Physiology of Hearing (Third Edition). Bingley: Emerald

[11] Ramamoorthy S, Deo NV, Grosh K (2007) A mechano - electro - acoutical model for the cochlea: resppnse to acoutic stimuli. J Acoust Soc Am 121: 2758-2773

[12] Salt AN (1995) Cochlear Fluid Research Laboratory. Available: http://oto2.wustl.edu/cochlea/. Last accessed 23th Feb 2014

[13] Thorne M, Salt AN, DeMott JE, Henson MM, Henson OW, Gewalt SL (1999) Cochlear fluid space dimensions for six species derived from reconstructions of three-dimensional magnetic resonance images. Laryngoscape 109:1661-1668 